\title{
Neuromuscular findings in thyroid dysfunction: a prospective clinical and electrodiagnostic study
}

Ruurd F Duyff, Joan Van den Bosch, D Martin Laman, Bert-Jan Potter van Loon, Wim H J P Linssen

\begin{abstract}
Objectives-To evaluate neuromuscular signs and symptoms in patients with newly diagnosed hypothyroidism and hyperthyroidism.

Methods-A prospective cohort study was performed in adult patients with newly diagnosed thyroid dysfunction. Patients were evaluated clinically with hand held dynamometry and with electrodiagnosis. The clinical features of weakness and sensory signs and the biochemical data were evaluated during treatment.

Results-In hypothyroid patients $79 \%$ had neuromuscular complaints, $38 \%$ had clinical weakness (manual muscle strength testing) in one or more muscle groups, $42 \%$ had signs of sensorimotor axonal neuropathy, and $29 \%$ had carpal tunnel syndrome. Serum creatine kinase did not correlate with weakness. After 1 year of treatment $13 \%$ of the patients still had weakness. In hyperthyroid patients $67 \%$ had neuromuscular symptoms, $62 \%$ had clinical weakness in at least one muscle group that correlated with FT4 concentrations, but not with serum CK. Nineteen per cent of the patients had sensory-motor axonal neuropathy and $0 \%$ had carpal tunnel syndrome. The neuromuscular signs developed rapidly, early in the course of the disorder and were severe, but resolved rapidly and completely during treatment (average time $\mathbf{3 . 6}$ months). Conclusions-Neuromuscular symptoms and signs were present in most patients. About $40 \%$ of the hypothyroid patients and $20 \%$ of the hyperthyroid patients had predominantly sensory signs of a sensorimotor axonal neuropathy early in the course of thyroid disease. Weakness in hyperthyroidism evolved rapidly at an early stage of the disorder and resolved completely during treatment, suggesting a functional muscle disorder. Hand held dynamometry is sensitive for the detection of weakness and for the clinical evaluation of treatment effects. Weakness in hypothyroidism is more difficult to treat, suggesting myopathy.

(F Neurol Neurosurg Psychiatry 2000;68:750-755)
\end{abstract}

Keywords: hypothyroidism; hyperthyroidism; myopathy; neuropathy; dynamometry

Correspondence to: Dr WHJP Linssen

Wim.Linssen@tip.nl

Received 6 September 1999 and in revised form

24 January 2000

Accepted 26 January 2000

Both hypothyroidism and hyperthyroidism may cause signs and symptoms of neuromuscular dysfunction. Hypothyroidism has been associated with the clinical features of myopa- thy (for example, proximal muscle weakness) ${ }^{1}$, mononeuropathy, and sensorimotor axonal polyneuropathy. ${ }^{2}$ Hyperthyroidism may cause myopathy $^{34}$ and possibly also polyneuropathy. ${ }^{5}$ The reported prevalence of these signs and symptoms is variable. Few prospective studies on this topic have been performed. ${ }^{7-10}$ In retrospective studies, published in the early $1980 \mathrm{~s},{ }^{11}$ the prevalence of neuropathy in hypothyroid patients varied between $10 \%$ and $70 \%$ and that of myopathy between $20 \%$ and $80 \%$, whereas the prevalence of myopathic features in hyperthyroidism varied between $60 \%$ and $80 \%$ of the patients. ${ }^{34}$

The aims of this study were (1) to investigate the prevalence of neuromuscular signs and symptoms in patients with newly diagnosed hypothyroidism or hyperthyroidism; (2) to evaluate electrodiagnostic evidence of neuromuscular dysfunction; (3) to study the effects of treatment; (4) to correlate the severity and duration of thyroid dysfunction with the serum creatine kinase (CK) concentration and the presence of neuropathy and myopathy; and (5) to compare the patterns of neuromuscular involvement between hypothyroidism and hyperthyroidism.

\section{Patients and methods}

PATIENTS

All adult patients with newly diagnosed thyroid dysfunction (abnormal serum concentrations of thyroid stimulating hormone (TSH) and free thyroxine (FT4)), who were seen at our internal medicine outpatient department between 1994 and 1997 were asked to participate in our prospective follow up study. A patient information leaflet was offered and informed consent was asked for. The study was approved by our local ethics committee.

Inclusion criteria were: (1) newly diagnosed hypothyroidism or hyperthyroidism that warranted treatment, and (2) older than 18 years. Exclusion criteria were: (1) other possible causes of neuropathy or neuromuscular diseases (for example, diabetes mellitus, alcoholism, liver and kidney disease, use of drugs known to cause neuropathy or myopathy, malignancy, or other serious illness (for example, cardiac failure or HIV infection), a family history of neuropathy), and (2) pre-existence of myopathy.

\section{CLINICAL EVALUATION}

For the neurological history a standardised symptom questionnaire was used with special attention given to sensory symptoms, weakness, cramps, muscle pain, fatiguability, difficulty climbing stairs, and rising from a low seat. 
Duration of symptoms was estimated in months before referral. Finally, the patients were asked whether neuromuscular symptoms, when present, were their first or main complaint(s).

The clinical neurological examination was performed by one experienced examiner (RFD), who was not blinded to the diagnosis hypothyroidism versus hyperthyroidism, because of the often evident clinical signs that suggested the diagnosis. The neurological examination consisted of the assessment of strength of 16 major muscle groups (neck flexors and extensors, shoulder elevators and abductors, elbow flexors and extensors, wrist flexors and extensors, hand grip; flexors, adductors and abductors of the hip, knee flexors and extensors, foot dorsi and plantar flexors) by manual muscle testing. The Medical Research Council (MRC) grading scale (0 to 5) for muscle strength was used. Summation of these MRC scores could lead to a maximum motor sum score of 150 .

The strength of 10 major muscle groups was quantified in a standardised way by means of a hand held dynamometer ( $\mathrm{HH}$ dynamometry) in Newtons. ${ }^{12}$ The dominant side of the patient was investigated. The $\mathrm{HH}$ dynamometer, with an $0.1 \%$ accuracy, was supplied by CITEC (Centre for Innovative Technics BV, Groningen, The Netherlands). The "break" technique was used and the average of three force measurements of each muscle group was calculated and compared with reference values. ${ }^{12}$ Weakness was defined as strength below the fifth percentile of strength in a normal population for each muscle group $\left(<\mathrm{P}_{5}\right) .^{12}$

The minimum rest period between measurements of one muscle group was 5 seconds. No warm up procedure before testing was used, because optimum warm up requirements for achieving stability of test data have not been identified. ${ }^{13}$ The position of the subjects during $\mathrm{HH}$ dynamometry were as described by Van der Ploeg et $a l,{ }^{12}$ with standardised joint angles and isometric contraction. Briefly, patients were firstly in the recumbent position when measuring force in the elbow flexors and extensors, hip flexors and abductors, knee flexors, and foot dorsiflexors. Subsequently they were seated in a chair for testing of neck flexors, shoulder abductors, wrist extensors, and three point grip. There was no visual feedback $^{14}$ (patients could not see the force value on the meter); all patients received similar verbal encouragement during testing.

The tendon reflexes were graded according to the Mayo Clinic scale of reflexes $(-4=a b s e n t$ to $+4=$ clonus). ${ }^{15}$ Sensory modalities (touch, pin prick, and vibration sense) were scored according to a proposed standard protocol. ${ }^{16}$ Summation of sensory grading scores could lead to a maximum sensory sum score of 48 (four point scales of three sensory modalities in all four limbs).

The height of all patients was measured at entry and their weight at all visits, and a body mass index (BMI) at each visit was calculated.
ELECTRODIAGNOSTIC EXAMINATION

The electrodiagnostic procedure was carried out according to standardised protocols. ${ }^{17}$ Before measuring the nerve conduction velocities (NCVs), distal limb skin temperature was measured and, when lower than $31^{\circ} \mathrm{C}$, the limb was warmed in a bath of water at $37^{\circ} \mathrm{C}$ for 30 minutes before testing. ${ }^{18}$

Concentric needle EMG was performed in the left deltoid, iliacus, supraspinatus, and thoracolumbar erector spinae and in the right brachioradial, abductor pollicis brevis, vastus medialis, gluteus medius, and anterior tibial muscles. The following concentric needle variables were scored: presence or absence of spontaneous muscle fibre activity (fibrillation potentials, positive sharp waves (in three grades), fasciculations, or complex repetitive discharges with gain setting at $50 \mu \mathrm{V} /$ division, evidence of reinnervation activity (polyphasic or giant motor unit action potentials (MUAPs)), and the discharge pattern on maximal voluntary effort (no, single, mixed, interference pattern). Amplitude and duration of motor unit potentials recorded during slight voluntary activation were noted. The MUAPs were considered to be "myopathic" when duration was less than $10 \mathrm{~ms}$. Whenever the qualitative visual analysis indicated abnormality, 20 MUAPs were photographed for detailed measurements. ${ }^{19} 20$

The nerve conduction studies consisted of the determination of the motor nerve conduction velocity (MNCV), amplitude, and duration of the compound muscle action potentials (CMAPs) after distal and proximal stimulation, and distal motor latencies (DMLs) of the median and peroneal nerves. The latency of the fastest $\mathrm{F}$ wave was recorded for the median and peroneal nerves. In addition, the sensory nerve conduction velocity (SNCV), amplitude, and duration of the sensory nerve action potential (SNAP) after distal stimulation of the median nerve, radial nerve, and sural nerve were studied. Latencies and amplitudes of Hoffman reflexes, and patellar and Achilles tendon reflexes were measured bilaterally. For the presentation of the results, the finding of small sized MUAPs with low amplitude and short duration was considered "myopathic", and the finding of decreased motor or sensory nerve conduction velocity with fibrillation potentials was considered "neurogenic".

Finally, the central motor conduction time (CMCT), with transcranial magnetic stimulation of the motor cortex ${ }^{21}$ (Magstim 200 with $10 \mathrm{~cm}$ circular coil), to the thenar and to the $\mathrm{m}$ extensor digitorum brevis (EDB) was calculated. The peripheral conduction time was calculated using the appropriate $\mathrm{F}$ wave and DML ((F+DML-1)/2). To obtain the CMCT, we subtracted this time from the latency of the muscle response found after cortical stimulation.

The neurological, dynamometric, and electrodiagnostic examinations were done at entry, when patients were still untreated. Neurological and dynamometric follow up was done after 3 to 4 months and after 1 year of therapy. At 
Table 1 Thyroid functions

\begin{tabular}{|c|c|c|c|c|}
\hline & \multicolumn{2}{|l|}{ Baseline } & \multicolumn{2}{|c|}{ Euthyroid state } \\
\hline & Hypothyroid & Hyperthyroid & Hypothyroid & Hyperthyroid \\
\hline $\begin{array}{l}\text { Mean TSH (mU/l) } \\
\text { Range }\end{array}$ & $\begin{array}{l}53 \\
(4.1-120)\end{array}$ & $<0.01$ & $\begin{array}{l}3.2 \\
(1.9-3.7)\end{array}$ & $<0.01$ \\
\hline Mean FT4 (pmol/1) & 6.1 & 67.1 & 15.7 & 19.1 \\
\hline Range & $(1.0-11.3)$ & $(33.4-128.4)$ & $(12.1-19.2)$ & $(12.8-22.1)$ \\
\hline Mean T3 (nmol/1) & 1.7 & 6.6 & 2.3 & 2.4 \\
\hline Range & $(0.6-2.6)$ & $(3.4-10)$ & $(1.0-2.4)$ & $(1.8-3.1)$ \\
\hline
\end{tabular}

that time all patients had been in the euthyroid state for at least 3 months.

LABORATORY INVESTIGATIONS

Serum concentrations of TSH, FT4, T3, CK, sodium, potassium, calcium, phosphorus, creatinine, glucose, vitamin B12, and folic acid were measured, together with autoantibodies against thyroid tissue; TSH, FT4, and T3 were monitored during treatment. Reference values of our laboratory for TSH are between 0.1 and $4.0 \mathrm{mU} / 1$, for FT4 between 12 and $24 \mathrm{pmol} / 1$, and for T3 1.3 and $2.7 \mathrm{nmol} / 1$ (Immulite, DPC, Los Angeles, USA).

\section{TREATMENT}

Directly after the initial neurological and neurophysiological examinations all patients received appropriate treatment for their thyroid disorder (for example, carbimazole, thyroxine, ${ }^{131}$ I therapy). Biochemical euthyroidism was reached within 17 weeks (mean, range 6-30 weeks) in hyperthyroid patients and in 19 weeks (mean, range 7-41 weeks) in hypothyroid patients. The mean TSH, FT4, and T3 values at baseline and during euthyroidism are presented in table 1 .

STATISTICAL ANALYSIS

When appropriate, Pearson's correlation coefficients were used. Paired or unpaired two tailed Student's $t$ tests were used after analysis of variance (ANOVA), to compare the means in the different subgroups. $p$ Values $<0.05$ were considered significant. In addition, the nonparametric Wilcoxon signed rank test and the $\chi^{2}$ test were used.

Table 2 Comparison of hypothyroid and hyperthyroid patients

\begin{tabular}{lccl}
\hline & $\begin{array}{c}\text { Hypothyroidism } \\
(n=24)\end{array}$ & $\begin{array}{c}\text { Hyperthyroidism } \\
(n=21)\end{array}$ & $p$ Value \\
\hline Age (y) (mean (SD)) & $47.8(13)$ & $43.2(13)$ & $\mathrm{NS}$ \\
Height (cm) (mean (SD)) & $165.1(5.1)$ & $167.5(8.2)$ & $\mathrm{NS}$ \\
Duration (months) (mean (SD)) & $20.8(15.5)$ & $7.3(4.3)$ & 0.006 \\
BMI at entry (mean (SD)) & $28.1(3.7)$ & $23.9(2.8)$ & $<0.005$ \\
BMI after therapy (mean (SD)) & $27.5(3.3)$ & $26.2(2.9)$ & $\mathrm{NS}$ \\
Symptoms (n (\%)): & $13(54)$ & $14(67)$ & $\mathrm{NS}$ \\
Weakness & $10(42)$ & $2(10)$ & $<0.05$ \\
Cramps & $19.9(14.7)$ & $4.4(2.5)$ & $<0.005$ \\
Duration (months) & $7(29)$ & $3(14)$ & $\mathrm{NS}$ \\
Sensory symptoms & $9(38)$ & $13(62)$ & 0.1 \\
Signs (n (\%)): & $0(0)$ & $16(76)$ & $<0.001$ \\
Paresis of muscle(s): & $1(4)$ & $9(43)$ & $<0.01$ \\
Tremor: & $16(67)$ & $4(19)$ & $<0.005$ \\
Ankle jerk >+1 & 147.9 & 141.8 & $<0.01$ \\
Ankle jerk <0 & 149.6 & 150 & $\mathrm{NS}$ \\
MRC sum 1st & 43.1 & 45.1 & $\mathrm{NS}$ \\
MRC sum 3rd & 45.8 & 46.8 & $\mathrm{NS}$ \\
Mean SSS 1st & $6.9(4.0)$ & $3.6(2.3)$ & $<0.05$ \\
Mean SSS 3rd & & & \\
Resolution of muscle symptoms (months) & & \\
$\quad$ (mean (SD)) & & &
\end{tabular}

$\mathrm{BMI}=$ body mass index; MRC sum 1 st and final=MRC sum score at initiation (1st) and after 1 year of therapy (final); Mean SSS 1st and final=mean sensory sum score at initiation (1st) and after 1 year of therapy (final); NS=not significant.

\section{Results}

In the study period 141 patients with untreated hyperthyroidism or hypothyroidism fulfilled the inclusion and exclusion criteria. Forty five patients (24 hypothyroid, 21 hyperthyroid) participated in the study. Thirty six patients were women $(80 \%)$. Most refusals were due to the prospect of undergoing electrodiagnostic examination, which would not contribute to further treatment.

The mean age of all participating patients was 45.7 years (range: 22-83 years; hypothyroid patients 47.8 years (SD 13.5), hyperthyroid patients 43.2 years (SD 12.9) (table 2)). The mean subjective duration of disease (at study entry) was significantly longer for patients with hypothyroidism: 20.8 months (range: 2-84), whereas for patients with hyperthyroidism it was 7.3 months (range: 2-24) $(p=0.006)$. Median durations were 12 and 6 months respectively. As expected, the mean BMI of hyperthyroid patients (23.9 (SD 2.8)) was lower than that of hypothyroid patients (28.1 (SD 3.7) $(\mathrm{p}<0.005))$. After 1 year of therapy this difference was no longer significant: 26.2 (SD 2.9) versus 27.5 (SD 3.3) $(p=0.3)$.

\section{HYPOTHYROIDISM}

Nineteen patients $(79 \%)$ had complaints suggestive of muscle dysfunction: weakness (54\%), fatiguability, muscle pain, stiffness, or cramps $(42 \%)$. The mean subjective duration of these symptoms was 19.9 months (range: $1.5-84$; median 12$)$. Only three patients $(16 \%)$ had muscle complaints as the presenting symptom or the main reason for consulting a doctor. Distally located sensory complaints (paraesthesiae or numbness) occurred in 29\% of the patients. Results are presented in table 2 .

\section{Clinical examination}

A total of $37.5 \%$ of patients had decreased muscle strength at manual muscle testing, especially of the proximal muscles (neck flexors, deltoideus, and iliopsoas). Weakness never exceeded grade 4 paresis. At $\mathrm{HH}$ dynamometry $58 \%$ of patients had muscle strength less than $\mathrm{P}_{5}$ of a normal population in at least one muscle group, ${ }^{12}$ whereas only $12.5 \%$ of all patients had more than four weak $\left(<\mathrm{P}_{5}\right)$ muscles. The duration of hypothyroidism was not significantly different between patients with weakness and patients with normal strength $(\mathrm{p}=0.5)$.

Symmetric distal sensory disturbances in the limbs (SSS <45) combined with depressed ankle tendon jerks - that is, clinical signs of a polyneuropathy-were found in $42 \%$ of the patients. A clinical carpal tunnel syndrome was found in $29 \%$ of the patients (often bilateral).

\section{Electrodiagnostic studies}

Short duration of MUAPs ("myopathic") were seen in $33 \%$, decreased nerve conduction velocity and fibrillation potentials ("neurogenic") in $17 \%$, and carpal tunnel syndrome in $25 \%$ of the patients. The CMCT was normal in all patients (to thenar: mean $6.3 \mathrm{~ms}$, to $\mathrm{m}$ extensor digitorum brevis: mean $11.8 \mathrm{~ms}$.). 
Table 3 Electrodiagnostic findings

\begin{tabular}{llll}
\hline & $\begin{array}{l}\text { Hypothyroidism } \\
(n=24)\end{array}$ & $\begin{array}{l}\text { Hyperthyroidism } \\
(n=21)\end{array}$ & $\begin{array}{l}\text { Reference and } p \\
\text { Values }\end{array}$ \\
\hline Peroneus MNCV & $48.3(3.4)$ & $48.6(3.4)$ & $>40 \mathrm{~m} / \mathrm{s} ; \mathrm{NS}$ \\
Median MNCV & $58.5(2.6)$ & $58.9(3.7)$ & $>49 \mathrm{~m} / \mathrm{s}, \mathrm{NS}$ \\
Sural SNCV & $46.5(4.5)$ & $45(3.6)$ & $>40 \mathrm{~m} / \mathrm{s} ; \mathrm{NS}$ \\
Radial SNCV & $53.9(6.3)$ & $54.2(6.3)$ & $>42 \mathrm{~m} / \mathrm{s} ; \mathrm{NS}$ \\
Median SNCV† & $43.9(6.5)$ & $48.3(4.3)$ & $>41 \mathrm{~m} / \mathrm{s} ; \mathrm{p}<0.05$ \\
ATR latency right & $35.1(2.3)$ & $35.7(2.4)$ & $<40.4 \mathrm{~ms}$ NS \\
ATR latency left & $35.2(2.4)$ & $35.7(2.3)$ & $<40.4 \mathrm{~ms}$ NS \\
PTR latency right & $20.1(1.4)$ & $20.2(1.3)$ & $<24 \mathrm{~ms}$ NS \\
PTR latency left & $19.9(1.2)$ & $20.1(1.1)$ & $<24 \mathrm{~ms}$ NS \\
H reflex soleus & $29.9(2.1)$ & $29.3(2.1)$ & $<34.4 \mathrm{~ms}$ NS \\
F response median & $26.5(1.4)$ & $26.4(1.9)$ & $<31 \mathrm{~ms} ; \mathrm{NS}$ \\
F response peroneal & $47.8(2.5)$ & $47.2(4.2)$ & $<61 \mathrm{~ms} ; \mathrm{NS}$ \\
CMCT thenar & $6.3(1.2)$ & $5.5(1.1)$ & $<8.0 \mathrm{~ms}$ NS \\
CMCT extensor digitorum brevis & $11.8(1.4)$ & $12.1(1.5)$ & $<20 \mathrm{~ms}$ NS \\
\hline
\end{tabular}

Values are means (SD)). ${ }^{\star}$ Hypothyroidism $(n=13)$; hyperthyroidism $(n=11) .+$ Hypothyroidism $(\mathrm{n}=16)$; hyperthyroidism $(\mathrm{n}=11) . \mathrm{MNCV}$ and $\mathrm{SNCV}=$ Motor and sensory nerve conduction velocity; ATR and PTR=Achilles and patellar tendon reflexes; Median and Peroneal=median and peroneal nerve. $\mathrm{H}$ reflex $=$ Hoffman reflex. $\mathrm{CMCT}=$ central motor conduction time. NS=not significant. (>): lower limit and $(<)$ upper limit of reference value. The reference latencies of tendon reflexes, $\mathrm{H}$ reflexes, $\mathrm{F}$ waves, and CMCT are directly related to age and height.

The mean results of the electrodiagnostic studies are given in table 3.

\section{Laboratory investigations}

The CK concentration was raised in seven patients. The mean CK (145 U/l, range 110-1542 U/1) was not significantly higher $(\mathrm{p}=0.06)$ in hypothyroidism than in hyperthyroidism. Serum CK did not correlate with weakness. No significant differences were found in T3, FT4, and TSH values between patients with and without weakness, and for patients with an increased serum CK concentration.

Follow up

During treatment muscle complaints resolved in $79 \%$ of the patients within an average time of 6.9 months (SD 4.0), median 5.5 months. As a consequence, after 1 year $21 \%$ of the patients still had complaints of weakness, which could be confirmed as clinical weakness (manual muscle testing) in $13 \%$ of the patients.

H H Dynamometry, excecuted after 1 year of therapy, showed a slight but significant overall (mean of 10 measurements for the whole group) percentual increase in muscle strength $(12.1 \%, \mathrm{p}<0.0001$, paired $t$ test). Two patients with residual complaints and paresis worsened and showed a small decrease $(6 \%, \mathrm{NS})$ in muscle strength after 1 year. The increase in strength was relevant, considering that parallel with clinical recovery, the mean BMI decreased by $2 \%$. Wrist extensors (19\%) and neck flexors $(17 \%)$ showed the largest increase in strength.

HYPERTHYROIDISM

At the initial examination 14 patients (67\%) had complaints possibly related to neuromuscular dysfunction: weakness (67\%), fatiguability, muscle pain, or cramps $(10 \%)$. The mean subjective duration of these symptoms (4.4 months (SD 2.5)) was significantly shorter in hyperthyroid than in hypothyroid patients $(\mathrm{p}<0.005)$. In five patients $(36 \%)$ the muscle symptoms were the main reason for consulting a doctor. Distal sensory complaints (numbness and paraesthesiae), occurred in three patients $(14 \%)$. Interestingly, these three patients showed prominent muscle weakness, a mean manual muscle testing sum score of 135 (maximum 150), and very high FT4 values (mean: 98.5 (SD 20) pmol/1), significantly higher than the FT4 of hyperthyroid patients without sensory complaints $(\mathrm{p}=0.01)$.

\section{Clinical examination}

Manual muscle testing showed decreased muscle strength in $62 \%$ of the patients, especially in the $\mathrm{m}$ iliopsoas and $\mathrm{m}$ quadriceps femoris. Weakness never exceeded grade 4 paresis. The mean manual muscle testing sum score was 141.8 for the whole group, which was significantly lower than the mean value in hypothyroid patients $(147.9 ; \mathrm{p}<0.01)$. Results are presented in table 2 .

At hand held dynamometry $81 \%$ of patients had weakness $\left(<\mathrm{P}_{5}\right)$ in at least one muscle group, ${ }^{12}$ whereas $48 \%$ of all patients had more than four weak muscles. The mean number of weak muscles/patient was 3.8 (SD 2.8) for the whole group (5.4 muscles in the $62 \%$ of patients with clinical evidence of paresis, and 1.1 in the $38 \%$ of patients without paresis). Hyperthyroid patients had a significantly larger number of weak muscles compared with hypothyroid patients (1.7 (SD 1.6); p<0.01).

The mean duration of hyperthyroidism was slightly but not significantly $(p=0.4)$ longer for the $(62 \%)$ group of patients with weakness $(8.2$ (SD 6.0) months), compared with the (38\%) group with normal strength (5.9 (SD 1.2) months). Also, the mean duration of muscle complaints did not differ significantly between these two subgroups $(p=0.8)$.

Symmetric distal sensory disturbances in the limbs combined with depressed ankle tendon jerks, clinically consistent with a polyneuropathy, were found in $19 \%$ of the patients. None of the patients had a clinical carpal tunnel syndrome. Generalised hyperreflexia (+2 or more) was seen in $38 \%$ of patients and tremor in $76 \%$.

Electrodiagnostic studies - "Myopathic" changes were seen in only $10 \%$, "neuropathic" changes in $24 \%$ of the patients. In two patients a (subclinical) sensory carpal tunnel syndrome was found (slight reduction of the median nerve SNCV). CMCTs to the thenar (mean: $5.5 \mathrm{~ms}$ ) and to the $\mathrm{m}$ extensor digitorum brevis (mean:12.1 ms) were normal. The mean results of the electrodiagnostics studied are presented in table 3 .

\section{Laboratory investigations}

The serum CK was normal in all hyperthyroid patients (mean CK: $21 \mathrm{U} / \mathrm{l}$ ) and did not correlate with weakness. The mean FT4 concentrations in patients with paresis (76.7 (SD 16.2) pmol/1) were significantly higher than the mean FT4 concentrations in patients without paresis (51.9 (SD 12.9) pmol/1; two tailed Student's $t$ test: $\mathrm{p}=0.02)$. The mean T3 concentrations were slightly but not significantly, higher (7.4 (SD 1.95) $\mathrm{pmol} / \mathrm{l} ; \mathrm{p}=0.25$ ) in the paresis group, compared with T3 (5.8 (SD 1.8) pmol/l) in patients with normal strength (see also table 1 ). 
Follow up

During treatment the complaints about weakness resolved in all patients within an average time of 3.6 months (SD 2.3); median: 2 months, which was shorter than the mean time to resolution of symptoms of hypothyroid patients (6.9 months, $\mathrm{p}<0.05$, two tailed $t$ test). Sensory complaints resolved after an average time of 6.8 months. By contrast with the hypothyroid group, none of the hyperthyroid patients had residual weakness of any muscle group at manual muscle testing after 1 year of treatment.

H H Dynamometry after 1 year of therapy showed a considerable and significant overall increase in muscle strength of $52 \%$ compared with the initial examination $(\mathrm{p}<0.0001$, paired $t$ test); patients with paresis showed a greater increase in strength $(64 \%)$ than non-paretic patients $(24 \%, \mathrm{p}<0.005)$. The neck flexors showed the largest increase in strength $(66 \%)$.

During treatment, the mean BMI of hyperthyroid patients increased by $11 \%$.

\section{Discussion}

This prospective cohort study shows that most patients with hypothyroidism and hyperthyroidism have muscle complaints (79 and $67 \%$, respectively). The complaints of weakness were confirmed by clinical testing in $37.5 \%$ of the hypothyroid patients and in $62 \%$ of the hyperthyroid patients. Cramps occurred more often in hypothyroid patients (table 2). HH Dynamometry showed weakness in at least one muscle group in $58 \%$ of hypothyroid patients and in $81 \%$ of those with hyperthyroidism. This suggests that $\mathrm{HH}$ dynamometry is more sensitive for the detection of weakness. Although $\mathrm{HH}$ dynamometry requires some expertise, it is more precise for the quantification of muscle strength ${ }^{22}{ }^{23}$ than MMT. Therefore, $\mathrm{HH}$ dynamometry has advantages for clinical follow up and for evaluating treatment effects.

In the literature ${ }^{1346724}$ the prevalence of neuromuscular disorders in thyroid dysfunctioning varies between $20 \%$ and $80 \%$. Most of these studies were retrospective and were carried out before modern sensitive FT4 and TSH assays were available. Our data invalidate the presumption that a high prevalence of neuromuscular symptoms in thyroid dysfunction is due to a late diagnosis.

A major shortcoming of this study is the lack of an age and sex matched control group. Unfortunately healthy control volunteers refused the burden of electrodiagnostic investigations. A selection bias may have occurred, because patients with muscle and/or sensory complaints could have given consent to the study more readily. Given this consideration lower prevalence data than those obtained in this study would be expected. Yet all studies cited in reviews for estimating prevalence data, have this shortcoming. ${ }^{24}$

Also, ethical considerations did not allow repeat electrodiagnostic studies in patients, which probably might have provided more objective data of the course of neuromuscular signs and symptoms in these patients.
ALL PATIENTS

Polyneuropathy associated with hypothyroidism has been described in many studies, ${ }^{12}$ but axonal neuropathy associated with hyperthyroidism seems rare $^{56}$ Our findings of a predominant sensory axonal neuropathy in $19 \%$ of the hyperthyroid patients is more than expected and indicates that neuropathy should also be considered in patients with hyperthyroidism.

Clinically and electrophysiologically the neuropathies in hypothyroidism and hyperthyroidism seem predominantly sensory, what is consistent with the finding that in newly diagnosed patients with thyroid disease the sensory symptoms and signs of a mixed sensorimotor neuropathy may predominate initially. When motor nerve conduction velocity is still within a normal range, the sensory nerve action potentials may be reduced at an early phase of the disease. ${ }^{25}$

For the diagnosis of muscle weakness, the serum CK concentration did not correlate with muscle weakness; neither did "myopathic" electrodiagnostic changes. Hence, for diagnosing muscle weakness in thyroid disease, these tests are not very helpful.

Measurements of CMCT were unrevealing for effects of thyroid hormones on the central motor pathways. In a study by Ozata et al (1996), ${ }^{26}$ abnormal CMCT results were found in a minority of hypothyroid and hyperthyroid patients. As the CMCT abnormalities did not normalise after treatment, the clinical relevance of these results is questionable.

\section{HYPOTHYROIDISM}

By contrast with the favourable prognosis in hyperthyroidism, 33\% of hypothyroid patients had residual symptoms and signs after 1 year of therapy. This may be explained by the pathological changes found in hypothyroid muscles: type II fibre atrophy, increased numbers of internal nuclei, and "core-like" structures in type I fibres. ${ }^{27}$ The absence of a significant correlation between the level of weakness and the biochemical severity of hypothyroidism, suggests that hypothyroid patients do have a myopathy rather than a functional muscle disease.

In our patients with hypothyroidism the prevalence of clinical and electrodiagnostically confirmed carpal tunnel syndrome was $25 \%$, whereas percentages varying from 2 to $20 \%$ are reported. ${ }^{1}$

\section{HYPERTHYROIDISM}

Although most studies have labelled muscle weakness in hyperthyroidism as myopathic, we question this opinion. We found symmetric proximal muscle weakness in $52 \%$ of the patients, which contrasted with electrodiagnosis showing no or only equivocal myopathic features, and normal serum CK. There is no specific pathological change in muscles of hyperthyroid patients and paraclinical evidence of myopathy is lacking. The muscle weakness in hyperthyroidism occurs often, evolves rapidly, and is severe; recovery is fast and good. Because of the significant correlation between 
severity of hyperthyroidism (expressed in FT4 values) and clinical muscle weakness (not reported before), it may be better to speak of a functional muscle disorder in hyperthyroidism. The increase in muscle strength outweighs the increase of the mean BMI.

Recently, carpal tunnel syndrome associated with hyperthyroidism has been described. ${ }^{28}$ The estimated incidence is at least $5 \%$. We found no clinical carpal tunnel syndrome in our group. In only two cases a subclinical sensory carpal tunnel syndrome was found, probably due to coincidence given the prevalence of (subclinical) carpal tunnel syndrome in the general population. ${ }^{29}$

We conclude that neuromuscular symptoms and signs occur in most newly diagnosed patients with thyroid disease. By contrast with the literature, predominant sensory signs of a sensorimotor axonal neuropathy occur in about $20 \%$ of the patients with hyperthyroidism. Weakness in hyperthyroidism may be due to a functional muscle disorder, rather than being a myopathy as seems to be the case in hypothyroidism.

We thank Dr H Schreuder and Professor JHJ Wokke for their help in initiating the study and Dr JAL Vanneste for his valuable comments.

1 Rao SN, Katiyar BC, Nair KRP, et al. Neuromuscular status in hypothyroidism. Acta Neurol Scand 1980;61:167-77.

Nemni R, Bottacchi E, Fazio R, et al. Polyneuropathy in hypothyroidism: clinical, electrophysiological and morphological findings in four cases. $\mathcal{F}$ Neurol Neurosurg Psychiatry 1987;50:1454-60.

3 Ramsay ID. Electromyography in thyrotoxicosis. $Q \mathcal{F ~ M e d}$ 1965;34:255-67.

4 Puvanendran K, Cheah JS, Naganathan N, et al. Thyrotoxic myopathy. A clinical and quantitative analytic electromyographic study. F Neurol Sci 1979;42:441-51.

5 Berlit P, Mahlberg U, Usadel KH. Zur Frage der Polyneuropathie bei Hyperthyreose - Eine klinischneurophysiologische Studie. Schweiz Arch Neurol Psych 1992;143:81-90.

6 Sözay S, Gökçe-Kutsal Y, Celiker R, et al. Neuroelectrophysiological evaluation of untreated hyperthyroid patients. physiological evaluation of unt

7 Khaleeli AA, Gohil K, McPhail G, et al. Muscle morphology and metabolism in hypothyroid myopathy: effects of treatment. F Clin Pathol 1983;36:519-26.
8 Khaleeli AA, Edwards RHT. Effect of treatment on skeletal muscle dysfunction in hypothyroidism. Clin Sci 1984;66: muscle

9 Nobrega ACLM, Vaisman CGS, Araujo CGS. Skeletal muscle function and body composition of patients with hyperthyroidism. Med Sci Sports Exerc 1997;29:175-80.

10 Olson BR, Klein I, Benner R, et al. Hyperthyroid myopathy and the response to treatment. Thyroid 1991;1:137-41.

11 Khaleeli AA, Griffith DG, Edwards RHT. The clinical presentation of hypothyroid myopathy and its relationship to abnormalities in structure and function of skeletal muscle. Clin Endocrinol 1983;19:365-76.

12 Van der Ploeg RJO, Fidler V, Oosterhuis HJGH. Hand-held dynamometry: reference values. 7 Neurol Neurosurg Psychiatry 1991;54:244-7.

13 Keating JL, Matyas TA. The influence of subject and test design on dynamometric measurements of extremity muscles. Phys Ther 1996;76:866-89.

14 Hald RD, Bottjen EJ. Effect of visual feedback on maximal and submaximal isokinetic test measurement of normal quadriceps and hamstrings. F Orthop Sports Phys Ther 1987;9:86-93.

15 Dale AJD, Kokmen E, Swanson JW, et al. Reflexes. In: Mayo clinic and Mayo foundation. Clinical examinations in neurology. St Louis: Mosby Year Book, 1991:240-54.

16 Notermans NC, Wokke JHJ, Franssen H, et al. Chronic idiopathic polyneuropathy presenting in middle or old age: a clinical and electrophysiological study of 75 patients. $f$ Neurol Neurosurg Psychiatry 1993;56:1066-71.

17 Kimura J. Electrodiagnosis in diseases of nerve and muscle: principles and practice. 2nd ed. Philadelphia: FA Davis, 1989:103-28.

18 Franssen H, Wieneke GH. Nerve conduction and temperature: necessary warming time. Muscle Nerve 1994; 17:336-44.

19 Wilbourn A. The electrodiagnostic examination with myopathies. F Clin Neurophysiol 1993;10:132-48.

20 Stålberg E, Falck B. The role of electromyography in neurology. Electroencephalogr Clin Neurophysiol 1997;103: 579-98.

21 Chiappa K. Transcranial motor evoked potentials. Electromyogr Clin Neurophysiol 1994;34:15-21.

22 Bohannon RW. Manual muscle test scores and dynamometer test scores of knee extension strength. Arch Phys Med Rehabil 1986;67:390-2.

23 Van der Ploeg RJO, Oosterhuis HJGH, Reuvekamp J. Measuring muscle strength. $\mathcal{F}$ Neurol 1984:231:200-3.

24 Klein I, Levey GS. Thyroid myopathy. Thyroid Today 1983; 6:1-6.

25 Donofrio PD, Albers JW. AAEM minimonograph 34: polyneuropathy: classification by nerve conduction studies and electromyography. Muscle Nerve 1990;13:889-903.

26 Ozata M, Ozkardes A, Dolu H, et al. Evaluation of central motor conduction in hypothyroid and hyperthyroid patients. F Endocrinol Invest 1996;19:670-7.

27 Ono S, Inouye K, Mannen T. Myopathology of hypothyroid myopathy. Some new observations. $\mathcal{F}$ Neurol Sci 1987:77: 237-48.

28 Roquer J, Cano JF. Carpal tunnel syndrome and hyperthyroidism. A prospective study. Acta Neurol Scand 1993;88: $149-52$

29 De Krom MCTFM, Knipschild TG, Kester ADM, et al. Carpal tunnel syndrome: prevalence in the general population. $\mathcal{F}$ Clin Epidemiol 1992;45:373-6. 